\title{
Long-Term Follow-Up Case of Multiple Retinal Arterial Macroaneurysms Developing Branch Retinal Vein Occlusion following Ruptured Macroaneurysm
}

\author{
Yuya Terubayashi Teruyo Kida Masanori Fukumoto Jun Sugasawa \\ Seita Morishita Hiroyuki Suzuki Tsunehiko Ikeda \\ Department of Ophthalmology, Osaka Medical College, Takatsuki, Japan
}

\section{Keywords}

Multiple retinal arterial macroaneurysms · Branch retinal vein occlusion · Macular edema

\begin{abstract}
Purpose: Retinal arterial macroaneurysm (RAM) has been reported in association with branch retinal vein occlusion (BRVO), and usually BRVO precedes RAM. We present a long-term follow-up case report of unilateral multiple RAMs that developed BRVO following ruptured RAM in the same retinal quadrant. Case Presentation: An 80-year-old woman presented with floaters in her right eye in June 2012. Visual acuity (VA) was 20/25 in her right eye with posterior capsular opacity. Her fundus showed the first ruptured RAM at the superotemporal vascular arcade with subinternal limiting membrane and subretinal hemorrhages not involving the macula. These were absorbed gradually with a VA of 20/20. After 2 years, the second RAM at the proximal superotemporal vascular arcade developed and impending BRVO occurred with macular edema at the distal site of the RAM. With the RAM located close to the arteriovenous crossing, her VA was dropped to 20/60. Intravitreal injection of ranibizumab was performed and macular edema was resolved with improved vision of $20 / 30$. Three months later, she realized a sudden vision loss of 2/200. Her posterior pole showed massive pre- and subretinal hemorrhages, and vitrectomy was performed. The source of bleeding was the third RAM's rupture in a different artery. Her vision improved to 20/30. The unaffected
\end{abstract}


Case Reports in
Ophthalmology

Case Rep Ophthalmol 2016;7:243-248

(C) 2016 The Author(s). Published by S. Karger AG, Basel www.karger.com/cop

Terubayashi et al: Long-Term Follow-Up Case of Multiple Retinal Arteria Macroaneurysms Developing Branch Retinal Vein Occlusion following Ruptured Macroaneurysm

eye showed no RAMs. Conclusion: We experienced a long-term follow-up case of multiple RAMs showing different courses. We should cautiously note that BRVO can occur following RAM at the arteriovenous crossing.

\section{Introduction}

Robertson [1] first reviewed retinal arterial macroaneurysm (RAM) in 1973. It usually occurs at the temporal retinal artery within the third bifurcation, and it is strongly associated with systemic hypertension and arteriosclerotic vascular changes [2]. The natural history is spontaneous involution; however, visual loss may occur secondary to macular edema, exudate, and hemorrhage [2]. In general, RAM presents as a single aneurysm, not multiple formation [3].

Aneurysms of the retinal arteries should be distinguished from those of capillaries and veins. Branch retinal vein occlusion (BRVO) also often occurs in patients with systemic hypertension, inducing capillary aneurysms at the terminal venous radicles. There is a wellknown association between BRVO and RAM; however, BRVO usually precedes the development of RAMs $[3,4]$.

We report on a long-term follow-up case of an 80-year old woman with multiple ruptured RAMs that showed different courses. In particular, one of the ruptured RAMs was located close to the arteriovenous crossing, and following the RAM, BRVO occurred with macular edema at the same retinal quadrant.

\section{Case Report}

An 80-year-old woman was referred with floaters in her right eye in June 2012. Visual acuity (VA) was 20/25 in her both eyes. She had a glaucoma attack in her left eye, and laser iridotomy had been performed more than 20 years ago. Following that procedure, she had cataract surgeries with intraocular lenses in both eyes. She takes eyedrops for glaucoma in her left eye and her intraocular pressure was $12 \mathrm{~mm} \mathrm{Hg}$ in both eyes under good control. Her right fundus showed a ruptured RAM at the superotemporal vascular arcade with subinternal limiting membrane and subretinal hemorrhages not involving the macula (fig. 1). Her blood pressure (BP) was 163/77 mm Hg, and she did not know that she had systemic hypertension. We recommended that she should be examined by an internist who diagnosed her with systemic hypertension and prescribed antihypertensive medications. Six weeks after initiation of a calcium antagonist, her BP decreased to around 140/75 mm Hg. Retinal hemorrhage was absorbed gradually by itself and her VA improved to $20 / 20$.

Two years after the first ruptured RAM, she was referred due to visual loss in her right eye. The second RAM at the proximal superotemporal vascular arcade developed and impending BRVO occurred with macular edema at the distal site of the RAM (fig. 2). Her VA had dropped to 20/60. Fluorescein angiography showed that this second RAM was located close to the arteriovenous crossing. Intravitreal injection of ranibizumab was performed for the macular edema. One month after anti-VEGF therapy, macular edema was resolved and her VA had improved to 20/30 in January 2015.

Five months later, she realized a sudden vision loss of $2 / 200$ in her right eye once again. Her posterior pole showed massive pre- and subretinal hemorrhages, and posterior capsular opacity also existed. Vitrectomy and posterior capsular opacity removal were then per- 


\section{Case Reports in Ophthalmology}

(C) 2016 The Author(s). Published by S. Karger AG, Basel www.karger.com/cop

Terubayashi et al: Long-Term Follow-Up Case of Multiple Retinal Arteria Macroaneurysms Developing Branch Retinal Vein Occlusion following Ruptured Macroaneurysm

formed. The source of bleeding was the rupture of the third RAM on a different artery (fig. 3 ). The fluorescein angiography showed no retinal vasculitis or exudates. Indocyanine green angiography was also performed. The unaffected left eye showed no RAMs. Her vision in the right eye improved to $20 / 30$, and there has been no recurrence to date.

\section{Discussion}

We experienced a long-term follow-up case of unilateral multiple RAMs showing different courses, and one of the ruptured RAMs induced BRVO. This case has yielded two interesting findings. First, she had three RAMs in her right eye and these RAMs ruptured differently and showed different courses. Generally, most RAM present as a single aneurysm, not multiple formation [3]. In her fluorescein angiography, there were no retinal vasculitis or exudates, such as idiopathic retinal vasculitis, aneurysms, and neuroretinitis, Eales' disease, Leber's miliary aneurysm, or Coats' disease [5, 6]. Second, the second ruptured RAM developed BRVO at the same retinal quadrant, and macular edema occurred. The RAM was located close to the arteriovenous crossing. The association between arterial macroaneurysm and BRVO is well known, and there are some papers reporting on RAM followed by BRVO [3, 7]. However, it is rare for BRVO to occur after a RAM rupture.

Macroaneurysms of the retinal artery are generally located within the first three orders of arterial bifurcation, and the natural tendency is to become fibrosed without any specific treatment [1]. Panton et al. [3] conducted a case-control study and reported that systemic hypertension was present in about $80 \%$ of patients with RAM. Meanwhile, $86 \%$ of RAM patients presented one macroaneurysm, while $12 \%$ had two. Interestingly, our case had three RAMs in one eye, and the second ruptured RAM induced BRVO and macular edema. Sometimes, for visual recovery, it is necessary to use the laser photocoagulation for RAM or surgical removal of the hemorrhage covering the macula. In this case, the fluorescein angiography showed that the second RAM was located close to the arteriovenous crossing. We did not perform the laser treatment to avoid retinal artery occlusion caused by the laser photocoagulation for RAM [8]. For the macular edema secondary to BRVO following the ruptured RAM, we treated the patient using an intravitreal injection of an anti-VEGF drug, ranibizumab. This treatment was effective in achieving a relatively rapid resolution of both the macular edema and the fibrosis of the RAM itself without obstruction of the artery. The patient recovered her vision. Our case involved a RAM close to the arteriovenous crossing; however, branch retinal artery occlusion did not occur following the ruptured RAM [9]. Some papers have reported that intravitreal bevacizumab or ranibizumab for RAM could be effective $[10,11]$. So far as we know, no paper has reported intravitreal injection of ranibizumab for macular edema concerned with BRVO following RAM located at the arteriovenous crossing. One case report discusses BRVO occurring 3 years after spontaneous obliteration of the RAM [12]. This case did not involve macular edema and resolved naturally. In our case, BRVO occurred much earlier, before the hemorrhage caused by the ruptured RAM was absorbed and induced macular edema.

Systemic hypertension can damage the blood vessels of the retina, e.g. arteriosclerosis, hemorrhages, cotton wool spots, and macular edema. Retinal vascular changes are associated with hypertension and can predict cardiovascular events. It is important to identify and treat systemic hypertension in order to reduce the risk of developing new ruptures of the RAM. In our case, we noticed the patient's systemic hypertension after the first RAM rup- 
tured. With this systemic hypertension under good control, there has been no recurrence to date.

In conclusion, we experienced a long-term follow-up case of multiple RAMs that showed different courses. Our case did not show retinal vasculitis during this long-term observation; however, there may be a possibility of a variant of idiopathic retinal vasculitis, aneurysms, and neuroretinitis. Our case demonstrates the importance of avoiding excessive blood pressure fluctuations. We should be careful to note that BRVO can occur following a RAM at the arteriovenous crossing.

\section{Statement of Ethics}

The authors have no ethical conflicts to disclose.

\section{Disclosure Statement}

The authors have no conflicts of interest to report.

\section{References}

1 Robertson DM: Macroaneurysms of the retinal arteries. Trans Am Acad Ophthalmol Otolaryngol 1973;77:55-67.

-2 Rabb MF, Gagliano DA, Teske MP: Retinal arterial macroaneurysms. Surv Ophthalmol 1988;33:73-96.

-3 Panton RW, Goldberg MF, Farber MD: Retinal arterial macroaneurysms: risk factors and natural history. Br J Ophthalmol 1990;74:595-600.

-4 Cousins SW, Flynn HW Jr, Clarkson JG: Macroaneurysms associated with retinal branch vein occlusion. Am J Ophthalmol 1990;109:567-570.

-5 Chang TS, Aylward GW, Davis JL, et al: Idiopathic retinal vasculitis, aneurysms, and neuro-retinitis. Retinal Vasculitis Study. Ophthalmology 1995;102:1089-1097.

6 Maggi C: Leber's retinal degeneration with miliary aneurysms. Am J Ophthalmol 1963;56:901-907.

7 Parodi MB, Da Pozzo S, Saviano S, Ravalico G: Branch retinal vein occlusion and macroaneurysms. Int Ophthalmol 1997;21:161-164.

8 Russell SR, Folk JC: Branch retinal artery occlusion after dye yellow photocoagulation of an arterial macroaneurysm. Am J Ophthalmol 1987;104:186-187.

-9 Mitamura Y, Miyano N, Suzuki Y, Ohtsuka K: Branch retinal artery occlusion associated with rupture of retinal arteriolar macroaneurysm on the optic disc. Jpn J Ophthalmol 2005;49:428-429.

10 Cho HJ, Rhee TK, Kim HS, et al: Intravitreal bevacizumab for symptomatic retinal arterial macroaneurysm. Am J Ophthalmol 2013;155:898-904.

-11 Wenkstern AR, Petersen H: Intravitreal ranibizumab in retinal macroaneurysm. Graefes Arch Clin Exp Ophthalmol 2010;248:1667-1670.

12 Battaglia Parodi M, Bondel E, Saviano S, Ravalico G: Branch retinal vein occlusion after spontaneous obliteration of retinal arterial macroaneurysm. Retina 1998;18:378-379. 


\section{Case Reports in Ophthalmology}

(C) 2016 The Author(s). Published by S. Karger AG, Basel www.karger.com/cop

Terubayashi et al: Long-Term Follow-Up Case of Multiple Retinal Arterial Macroaneurysms Developing Branch Retinal Vein Occlusion following Ruptured Macroaneurysm

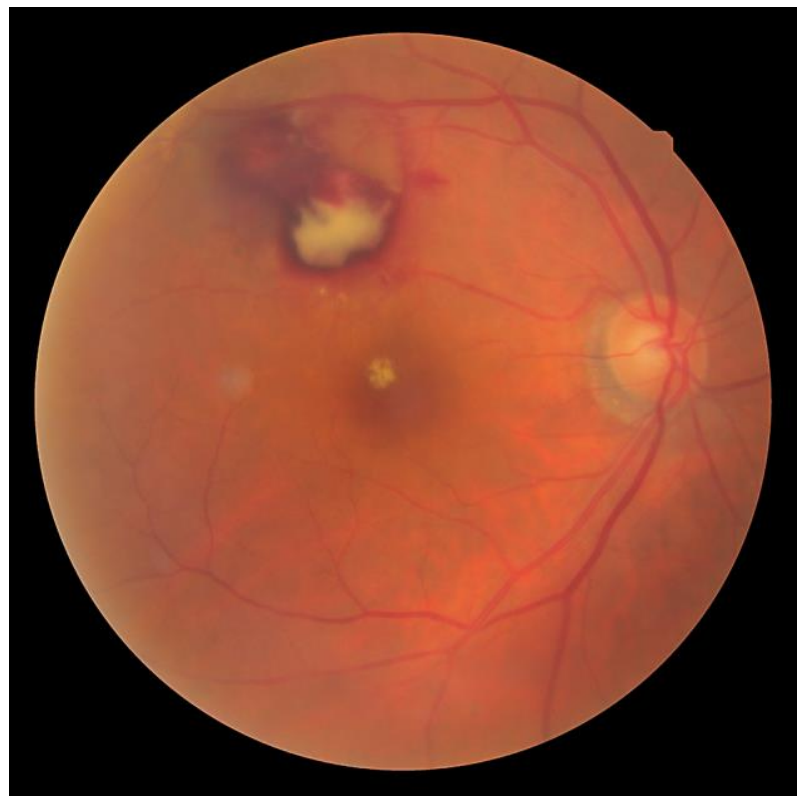

Fig. 1. Her right fundus showed the first ruptured RAM at the superotemporal vascular arcade with subinternal limiting membrane and subretinal hemorrhages not involving the macula. During her first visit, her BP was $163 / 77$ mm Hg with no medications. Her VA was 20/25.

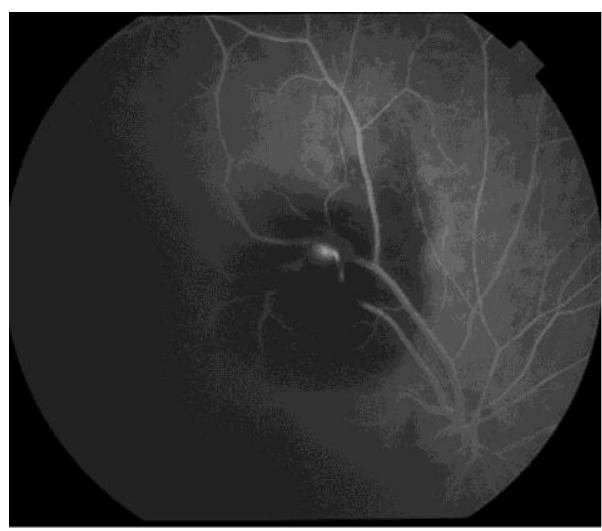

$30 \mathrm{sec}$.

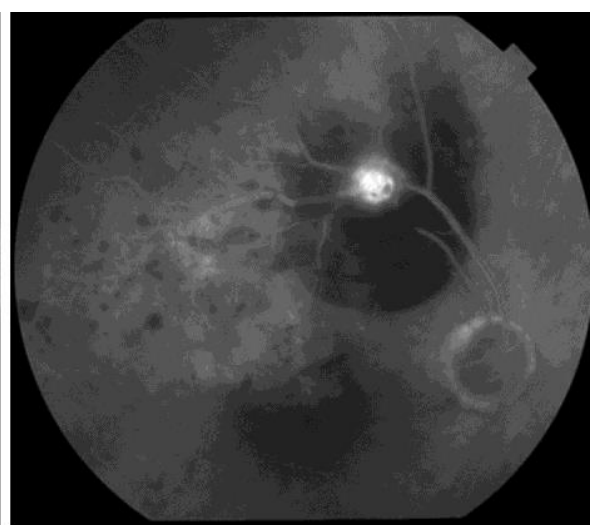

$8 \mathrm{~min}$.

Fig. 2. The fluorescein angiography showed that the second ruptured RAM was located close to the arteriovenous crossing and BRVO existed at the distal site of the RAM. There was fluorescein leakage in the macula. Her VA had dropped to $20 / 60$. 
Case Reports in
Ophthalmology

Case Rep Ophthalmol 2016;7:243-248

(C) 2016 The Author(s). Published by S. Karger AG, Basel www.karger.com/cop

Terubayashi et al: Long-Term Follow-Up Case of Multiple Retinal Arterial Macroaneurysms Developing Branch Retinal Vein Occlusion following Ruptured Macroaneurysm
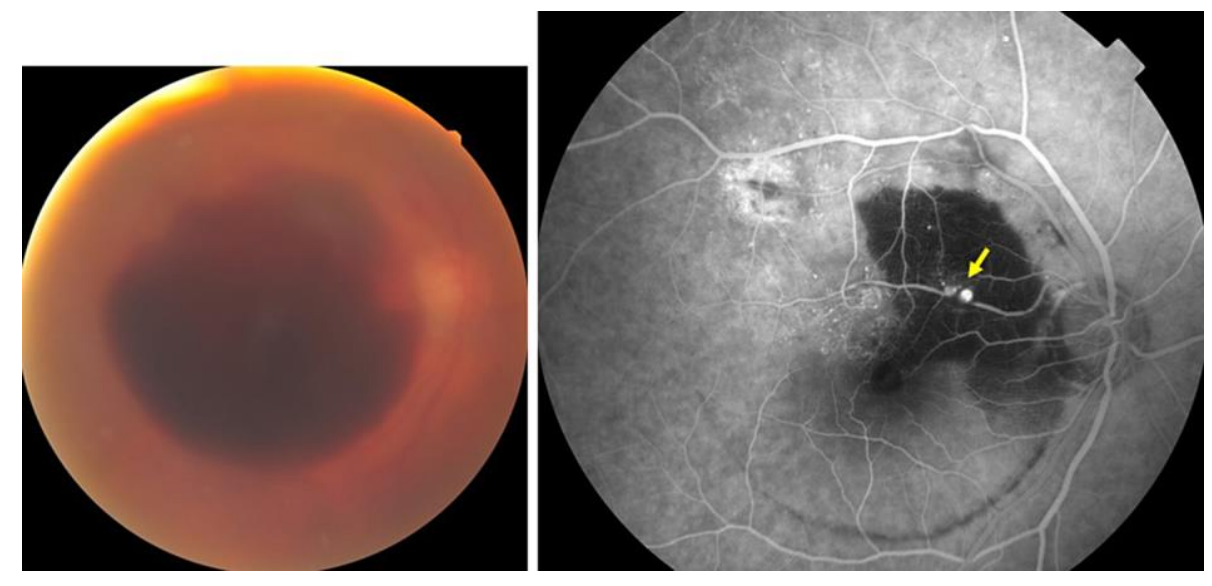

Fig. 3. The third RAM ruptured (arrow) and her posterior pole showed massive pre- and subretinal hemorrhages. Her VA was 2/200 in her right eye. We performed fluorescein angiography 11 days after vitrectomy. 